\title{
THE PATHOLOGY
}

of

\section{PARALYSIS WITH MUSCULAR DEGENERATION (PARALYSIE MYOSCLEROTIQUE), OR PARA- LYSIS WITH APPARENT HYPERTROPHY.}

\author{
By Dr. DUCHENNE of Boulogne.
}

[Abstract of a cammunication made to the Pathological Society of London by Mr. Lockhart:Clarke, F.R.S., in the name of the author.]

I BRING before your notice this evening a paper with which, during a recent visit to Paris, I was entrusted by Dr. Duchenne, for the purpose of communicating it in his name to this Society. I have carefully translated the paper, and have endeavoured to preserve every shade of the author's meaning. He calls the disease of which it treats by the name of "Paralysie Myosclérotique", or "Paralysie avec Hypertrophie Apparente"; that is, "Paralysis with Muscular Degeneration", or "Paralysis with Apparent Hypertrophy". The term "sclerosis" was first used by the Germans to signify a degeneration of tissue, and is used by the French in the same sense. But the application of the term is not correct; since the Greek word from which the term sclerosis is derived indicates a process of hardening, whereas the term sclerosis is applied to cases in which, as in ataxy, etc., there is not necessarily any induration of tissue.

\section{Summary of Symptomology.}

The clinical facts which serve as a basis for studying the symptomology of the disease show that paralysis with degeneration (sclerosie) of muscles, or with apparent hypertrophy of muscles, is marked, in general, by three distinct periods or stages-a stage of paralysis, a stage of hypertrophy, and a stage in which the paralysis becomes general.

I. The first stage is manifested either at the time when the children should begin to walk, or some years after they have begun. In the former case, although the conformation of these children be quite normal, and although, while lying down or in the arms of their mothers or their nurses, they appear to possess their natural share of motility, yet, when they arrive at the age of twelve or fourteen months, if an attempt be made to stand them on their legs, they immediately fall down. It is not till they have attained the age of two or three years, that they are able to stand upright or to walk, and even then they require support. In the latter case, after these children have walked well for several years, it is remarked that, either spontaneously or subsequently to some convulsions, they are soon fatigued by standing or walking; that, without some support, they find these operations become more and more difficult and painful, and that they are subject to frequent falls. Whatever may have been the age at which the malady first made its appearance, it is soon observed that, in order to maintain their equilibrium while standing or walking, all these children bend themselves very much backward and keep their legs very much apart; that at each step they incline laterally towards the leg which rests on the ground-a movement which produces a characteristic balancing of the body during progression.

2. The second stage is announced, in general, some months, and even two years, after the beginning of the muscular weakness, by a progressive swelling or enlargement of the gastrocnemii, then of the glutei and the lumbar muscles of the spine. This apparent hypertrophy occurs sometimes in nearly all the muscles that have been affected by paralysis; but, in general, it does not, and it may even be limited to a very small number of the muscles. The extension, to a greater or less extent, of the apparent hypertrophy of the muscles may constitute different varieties of this kind of paralysis. The hypertrophied muscles are firm and elastic; they become very hard while they contract, and show all the relief or projection which properly belongs to their contracted state; they then appear to form a hernial protrusion through the integument, which is very thin. Moreover, their great size shows off the apparent smallness and delicacy of the joints at the knee, ankle, etc.

In one case that came under my observation, both the weakness and the muscular hypertrophy appear to have shown themselves simultaneously. According to the information given by the mother, the child was very large at its birth. But this information is insufficient; we ought to know whether the great size of the body and of the limbs was or was not due rather to the abundance of subcutaneous adipose tissue than to the volume of the muscular masses.

The increase in the size of the muscles does not appear to add to their weakness. This is so far from being the case, that the muscles of the calves, which are always the most hypertrophied, are those which are found to have relatively the greatest power.

The morbid phenomena above described may remain in the same state for years-sometimes until a tolerably advanced period of youth.

A new stage of the disease, and the last one, is manifested by a gradual increase in the severity, and a more general extension, of the paralysis. The young patients can no longer stand upright; they always remain in the recumbent posture without any power to change the position in which they may be placed; and the upper extremities, if they have not bitherto been affected, soon lose all their movements. With this aggravation of the paralysis, hypertrophied muscles may sometimes be seen to melt, as it were, away, and then all the limbs and the trunk become atrophied en masse. Although in this stage the patients are reduced to a state of great weakness; they may nevertheless live for a tolerably long time. They are cut off by some intercurrent disease.

The figures whith accompany this paper represent the appearances of several children differently affected by this disease. Of these cases, by far the most remarkable was a boy whom I saw in the Hopital St. Eugénie, under the care of Dr. Bergeron. Every visible muscle of the body, except the pectorals, was enormously developed. When he walked, the poor boy had a most ludicrous appearance.

\section{Cases of Different Varieties of Apparent Hypertrophy in Paralysis with Degeneration of Muscles.}

CASE I.-A boy, aged 9, was attacked by paralysis with degeneration of muscles. The volume of the gastrocnemii increased enormously a few months after the first appearance of the paralysis of the inferior extremities. The glutei and the lumbar muscles of the spine at the same time increased in size, but in a less degree. He had curving of the back while standing upright or walking; separation of the legs, and lateral deviation of the trunk during progression. There was persistence of the same condition in spite of Faradisation (which showed that the electro-muscular contractility was intact) and hydrotherapy. This patient, who was first seen in $186 \mathrm{I}$, in the private practice of Dr. Duchenne (de Boulogne), changed his residence in 1864 . His neighbours state that, a year before he left, he was constantly confined to his bed; and that the paralysis had reached his upper extremities.

CASE II.-A boy, aged 8 years, was attacked by paralysis with degeneration of muscles at about the age of six years. All the muscles of the inferior members, the glutei, and the lumbar muscles of the spine, acquired progressively a considerable size about a year after the weakness of the upper extremities began. This apparent hypertrophy of the muscles contrasted strongly with the emaciation of the upper extremities and trunk. The great curve in the back which is seen in this case always exists in this stage of the disease. This condition remained stationary for several years, after which the paralysis reached the upper extremities, and became general. The patient died in 1866 , at the age of $14 .^{*}$

CASE III.-A boy, aged II years, was affected with paralysis and muscular degeneration from his earliest infancy. The apparent hypertrophy of the muscles is general, and gives to the limbs and body of the boy an athletic appearance. He is now at the Hospital St. Eugénie. Dr. Duchenne obtained, by means of his histological emporte-pièce, small portions of the muscles, in which the microscope showed the existence of hypertrophy of the interstitial connective tissue, with a tolerably abundant formation of fibrous tissue. A piece from one of the calves of the legs of the same subject, obtained by means of the emporte-pièce on October 25 th, 1867 , was prepared and examined by Mr. Lockhart Clarke, who has kindly done M. Duchenne the honour of communicating to the Pathological Society a resumé of his work on Paralysis with Muscular Degeneration.

CASE IV.-A boy, aged $7 \frac{1}{2}$ years, who began to walk very late, was afflicted with the same disease. His intellect was dull, and he had muscular weakness from his earliest infancy. At the age of four years, there was very evident increase in the size of his calves, of the glutei and the lumbar muscles of the spine, but in a less degree than in the preceding cases. The symptoms and general course of the malady, however, were the same. Thus, while walking or stand. ing, there was great curving of the loins, separation, of the inferior extremities, and lateral bending of the trunk at each step. Then, after remaining for some years stationary, the paralysis veached the upper

* This case was described in detail by. Dr. Duchenne, in the second edition of his work on L'electrisation localisee, p. 203. It was used for drawing a picture of this disease, which he then called paraplégie hypertrophique de l'enfance. 
extremities, and abolished all movement. He died in 1865 , at the age of thirteen years. He first came under observation in 1860, in Dr. Duchenne's private practice.

Dr. Duchenne informs me that he has been studying this disease for the last eleven years, and that he is now preparing a small work on the subject. Several cases have been recorded on the Continent, and post mortem examinations have been made. Eulenberg and Cohnheim examined the body of a child whicb died of this disease at the age of thirteen. They found the electro-muscular contractility everywhere intact. Nothing abnormal was discovered in the nervous and vascular systems. To the touch, the muscles of the lower limbs gave the sensation of a doughy and inelastic mass. They were marked with stripes or striæ of a yellow or yellowish white appearance. On section, they shone with a kind of greasy light. 'At certain points, they could not be distinguished by the naked eye from the subcutaneous adipose tissue. The muscles of the upper extremities presented a similar kind of structure; but they were much atrophied, as were also those of the trunk. Under the microscope, those especially of the lower extremities seemed to be filled with adipose tissue; but the muscular tissue itself was not altered. Griesinger and Billroth had already observed a similar state in the living subject. It is rare, however, to find any oily particles in them. (Ver. handl. d. Berliner Med. Ges., 1, 101-205.) Heller, who examined two brothers that died of this disease, seems to consider it as a kind of fatty degeneration, for he calls it lipomatous. (Deutsche Archiv Klin. Med., r, 6r6-627.) Seidel also records three cases belonging to the same family, under the term of "lipomatous atrophy of the muscles, or muscular atrophy."

By means of an ingenious instrument which he has himself invented, Dr. Duchenne has been able to obtain small portions of the muscles from different parts of the boy whose appearance is represented in Figs. 5 and 6 . In these specimens of muscular tissue, when examined under the microscope, Dr. Duchenne discovered evident hypertrophy of the interstitial connective tissue, with a rather abundant formation of wavy fibrous tissue. In a piece which he removed in my presence from one of the calves of the same boy, I found a similar increase of fibrous and connective tissue; and I also found, what I had not seen in Dr. Duchenne's preparation, large collections of adipose tissue between bundles of muscular fibre. The muscular fibre itself, however, appeared to be unaffected; and, although numerous oil-globules were scattered here and there, they resulted, I believe, from rupture of the fat-cells; for they were not incorporated with the muscular fibres, as in the case of fatty degeneration.

The ingenious contrivance which Dr. Duchenne has invented for obtaining portions of muscle from the living subject, and which he calls his emporte-pièce, is very superior to the harpoon or hook invented by Middeldorf, and employed in Germany. It consists of a steel cylindrical rod, divisible longitudinally into two halves. One of these is fixed to a handle by means of a screw, and terminates at the free end in a small hollow cone, the base or lower border of which has a sharply-cutting edge. When closed, the two pieces together form a small cylindrical rod with a fine point. As soon as it is made in this state to penetrate a muscle, the hollow cone is opened by sliding down the other half of the rod, and encloses a small portion of the muscle, which it cuts away by means of its sharp edge.

\section{ON THE USE OF THE VECTIS IN ASSISTING PARTURITION.}

By G. D. R. MCCARTHY, Esq., Wrockwardine Wood, Wellington, Salop.

IT has long been my opinion that there is great danger in delaying interference in protracted difficult cases; not only where there is unmistakable mechanical obstruction (pelvic), but also where there is atony or spasmodic action of the uterus, or general debility and sinking, or delay from some cause which cannot be exactly known. I have seen cases where the progress of the labour has been unaccountably arrested. In these circumstances, shall we wait for Nature to act-or, as it is generally expressed, shall we "leave it to Nature"? There is no doubt that some cases thus left to Nature do well. But some do not; and amongst such I include not only those very few patients who die undelivered, but those whose vital powers have been so worn out by long, tedious, ineffectual efforts that they sink shortly after delivery, or make an exceedingly slow recovery; and those also who have experienced actual lesion of the uterus or neighbouring parts, or violent action of the whole system, issuing in peritonitic affection, pyæmia, puerperal mania, etc. I am quite sure that too much homage is paid to this power we call Nature. It is well known that, if she were not very often at a fault, we should have nothing wrong, or, if wrong, it would be invariably set right; but, as she is, it becomes us, with all due care and assiduity, to render her all the help we can.

With such views as these, $I$ have-acted in difficult and in most protracted midwifery cases. As to ergot, it is certainly a valuable medicine, both for assisting the expulsive efforts and for controlling after-hæmorrhage. But, if given too early, it is worse than useless. I cannot say I have often known it injure the foetus; but it must be obvious to every reasonable mind that, where there is considerable mechanical obstruction to be overeome (as in case of rigidity of the os uteri or in malposition of the head); and indeed until the os is very nearly dilated and the head at least half engaged in the pelvis, ergot is dangerous. The os may suddenly yield, the head may easily descend, and all be over just to our mind; but it may not; and then all the ineffectual efforts caused by the ergot will have contributed to make the case more difficult and inauspicious. But, when the os is pretty freely dilated, if there be much delay and the pains be weak and variable, and the patient's strength becoming less, I have not hesitated to have recourse to instrumental means. For some years I used the forceps, but for many years I have greatly preferred the vectis, and have found it a far more manageable instrument; and preferable for the following reasons.

I. It may be introduced with perfect ease, and frequently hardly perceived by the patient.

2. It produces but slight marks on the foetal head.

3. It is available before the head is low enough for the forceps; and can therefore assist in the most difficult stage of the labour, the passage between the bones.

4. It renders assistance unnecessary, which would be almost indispensable in applying the forceps to retain the first blade in its place while adjusting the other.

5. It does not alarm the patient or her friends by the clicking of "those things". For, if they know it at all, it may be described as a single instrument of great simplicity and harmlessness.

6. It may be easily shifted from one side of the pelvis to the other.

7. There is very little danger of its causing laceration of the perinæum.

8. It is more portable than the forceps, and may therefore be generally carried in the pocket, and thus render needless the awful ceremony of sending home for instruments.

In all the above particulars, I have found the vectis the best instrument. For if, after all, it cannot extract the head when it has descended into the lower region of the pelvis, the forceps can but be used, and would require but little trouble to apply them. It has nearly superseded the use of the forceps in my practice. Not entirely; for occasionally one meets with a case of impaction which calls for the forceps or even the perforator; though I am thankful to say I have not met with many such. I consider it as a sort of artificial hand. An accoucheur must often wish he could introduce his hand so as to pass it behind the head and help it in its passage. Now this desideratum is in a great measure potentially, though not actually, secured by the vectis in the manner in which $I$ have used it.

My method is to introduce the four fingers of my left hand, well anointed with lard, with their backs to the perinæum, and, bringing the handle of the instrument* to the pubes, the thighs being held open, slide the convexity of the curve along the inside of my fingers and then between the sacrum and the head, which is often done in a minute or two. I then bring the handle to a line in the direction of the upper axis of the pelvis, and, first depressing it-the patient lying at the edge of the bed - and then gently elevating it, the curved part passes over the head. Then, holding the handle steadily with my left hand, and carefully maintaining a sustained traction, examining with my right the progress, I keep the head from receding in the interval of the pains, which, as a proof how glad-if I may so express it-Nature seems of seasonable assistance, become more powerful and effectual. If I do not gain anything, after a short time, I partially withdraw the instrument and introduce it on the lower side of the head, when it lifts it up like a lever, using some degree of traction at the same time. If I find I cannot slide the instrument over the head as I wish, I withdraw it for a time and then introduce it again, when it generally succeeds. Under these circumstances, I give the ergot in decoction, and thus gain all the natural and artificial combined power possible.

I am happy to say that I have greatly facilitated and accelerated the delivery of many by this simple instrument, using it, as I have said, like a hand, and never injured the mother, and seldom the child. I believe it requires a little tact, which may be gained by practice; and whoever has tried it somewhat frequently-I mean as often as the protracted nature of cases, or the failing of natural efforts, have caused alarm and anxiety to any degree-has, I dare say, experienced its usefulness. I beg leave to offer the foregoing statements and observations to the consideration of my obstetrical brethren.

* I use one that shuts together and fastens open with a small button that turns. 